International Journal of Neuroscience Research
(ISSN:2572-8385)

\title{
Environmental Enrichment Reduces Cortical Excitability In Rats Submitted To Status Epilepticus In The Pilocarpine Model: An Evaluation From Cortical Spreading Depression
}

Roberto Carlos da Silva Bandeira', Luís Gustavo Carvalho dos Santos², Christian Barker Silva Santisteban ${ }^{2}$, Angela Amancio-dos-Santos ${ }^{3}$

${ }^{1}$ Federal University of Pernambuco - UFPE, Health Sciences Center, Department of Pharmaceutical Sciences, Recife - PE, Brazil; ${ }^{2}$ Federal University of Pernambuco - UFPE, Medical Sciences Center, Recife - PE, Brazil; ${ }^{3}$ Federal University of Pernambuco - UFPE, Biosciences Center, Department of Physiology and Pharmacology, Recife - PE, Brazil

\section{ABSTRACT}

Objective: This study verified if the exposure to environmental enrichment (EE) influences brain excitability, evaluated by

${ }^{*}$ Correspondence to Author: cortical spreading depression (CSD), in rats submitted to status Federal University of Pernambuco epilepticus (SE). Methods: At 35 days of age, male Wistar rats - UFPE, Health Sciences Center, were divided into two experimental groups: pilocarpine (PILO), Department of Pharmaceutical Sciwhich received a single dose of pilocarpine hydrochloride to ences, Recife - PE, Brazil; induce SE, and saline (SAL), which were the group control. On the following day, half of the animals in each group were exposed to EE; while the other half was kept in the standard environment (S), considered control. At 67 days of age, the rats were anesthetized and submitted to CSD. Results: Pilocarpine, isolated, reduced the speed of propagation and increased the duration and amplitude of CSD. EE, itself, reduced the speed of propagation and duration, but increased the amplitude of CSD. In the animals exposed to EE that undergone SE, the values of CSD parameters became closer to the control group. Pic Conclusion: Our results indicate that EE reduced the cortical From Cortical Spreading Depresexcitability induced by SE, possibly by promoting antioxidant sion. International Journal of Neueffects at the brain level, inducing neurogenesis and/or improving roscience Research, 2021; 5:13. the excitatory/inhibitory balance of neurotransmitters. Keywords: epilepsy. cortical spreading depression. pilocarpine.

How to cite this article:

Roberto Carlos da Silva Bandeira, Luís Gustavo Carvalho dos Santos, Christian Barker Silva Santisteban, Angela Amancio-dos-Santos. Environmental Enrichment Reduces Cortical Excitability In Rats Submitted To Status Epilepticus In The Pilocarpine Model: An Evaluation roscience Research, 2021; 5:13. eScîPUb
eSciPub LLC, Houston, TX USA.
Website: https://escipub.com/ 


\section{INTRODUCTION}

Environmental enrichment (EE) is a condition that mimetizes an active lifestyle at the laboratory level. It refers to living conditions in larger cages, with the inclusion of inanimate objects of various textures, sizes and shapes. This results in increased sensory, cognitive and motor stimulation, compared to living conditions in standard cages ${ }^{1}$. It has been reported that $E E$ increases brain plasticity, improves the metabolism of the nervous system, as well as cognitive and behavioral parameters ${ }^{1,2,3}$. Recent research from our laboratory has shown that environmental enrichment reduces the propagation of cortical spreading depression $(\mathrm{CSD})^{4}$.

Epilepsy is a brain disorder caused by the permanent predisposition of the brain to generate spontaneous and recurrent seizures accompanied by neurobiological, cognitive and social consequences ${ }^{5}$. Among the types of epilepsy, temporal lobe epilepsy (TLE) has great clinical relevance due to its high incidence and severity and it's often refractory to current drug therapy. Thus, unconventional therapeutic strategies, such as EE, could contribute to reduce the sequelae of this condition. One of the experimental ways to induce TLE in rats is through the pilocarpine model ${ }^{6}$.

Pilocarpine is an alkaloid extracted from the plant Pilocarpus jaborandiand and is a potent muscarinic cholinergic agonist. When administered systemically in high doses, it's capable of generating brain changes, both at the morphological level and at the neurochemical level, known as status epilepticus (SE); which is characterized by repeated seizures without complete recovery of neurological functions or continuous behavioral and/or electrical seizures $^{6,7}$.

CSD is an electrophysiological phenomenon that has been implicated in the mechanism of serious diseases, such as epilepsy, migraine with aura and cerebral ischemia ${ }^{8.9}$. This phenomenon was discovered in 1944 by
Aristides Leão, and is characterized by a marked reduction (depression) in the amplitude of spontaneous electrical activity observed in the electrocorticogram (ECoG), after electrical, mechanical or chemical stimuli. This depression propagates (spreads) in a concentric way, from the stimulated point, with speeds ranging from 2 to $5 \mathrm{~mm} / \mathrm{min}^{8}$. It's a reversible phenomenon and has been recorded in neuronal populations of several species.

It has been documented that EE has significantly reduced the number and duration of seizure episodes in epileptic rats ${ }^{10}$. Thus, it is possible that the EE to restore the excitation/inhibition balance in the brain. However, until now, there are no studies that corroborate this. It is believed that EE is a low-cost, affordable approach that could benefit the treatment of epilepsies. So, the objective is to verify whether exposure to environmental enrichment influences brain excitability, being assessed based on the parameters of cortical spreading depression, in rats that were submitted to status epilepticus.

\section{METHODS}

This study was submitted and approved by the Ethics Committee on the Use of Animals of the Federal University of Pernambuco (UFPE, Brazil; process number 23076.002253/2019-58) and is in accordance with the principles adopted by the Brazilian College of Animal Experimentation (COBEA) and with international standards established by the National Institute of Health Guide for Care and Use of Laboratory Animals.

Male, young rats, of the lineage Wistar, from the colony of the bioterium of the Department of Physiology and Pharmacology of UFPE were used. The animals were kept in standard bioterium conditions, in a room at a temperature of $22 \pm 2{ }^{\circ} \mathrm{C}$, submitted to an artificial light-dark cycle of $12 / 12$ hours (the dark beginning at seventeen hours), with free access to water and food (commercial diet).

At 35 days of age, two experimental groups were formed: 
1) Pilocarpine (PILO; $n=13$ ) - the animals received a single dose of pilocarpine hydrochloride $(200 \mathrm{mg} / \mathrm{kg}$, i.p.). In the 30 minutes prior to the procedure, a dose of methyl scopolamine bromide (1 $\mathrm{mg} / \mathrm{kg}$, i.p.) was administered to inhibit the peripheral action of pilocarpine. Two hours after the beginning of the $\mathrm{SE}$, a dose of diazepam (10 mg/kg, i.p.) was administered in order to reduce animal mortality. In the following 10 minutes, the animals received a simplified Ringer (saline) injection for volume replacement. In the two to three days after this procedure, the animals received special care and had volume replacement with simplified Ringer's solution when necessary. This SE induction protocol follows the standards described in the literature.

2) Saline (SAL; $n=13)$ - the animals received a simplified Ringer's solution (saline), a vehicle solution for pilocarpine. The saline was administered at a dose of $10 \mathrm{~mL} / \mathrm{kg}$, i.p.. These animals were considered as the control rats of the pilocarpine group.

The day after the administration of pilocarpine or saline, at 36 days of age, the animals were distributed into the following groups:

1) Environmental Enrichment (EE) - the animals were housed (in groups of 4 animals), daily, for three hours (start at $7 \mathrm{am}$ ), for four weeks, in an acrylic cage, with dimensions of $100 \times 60 \times 35$ $\mathrm{cm}$. The cage had several objects, such as toys, wheels, plastic tubes of different diameters and others. To ensure the presence of a new component, the configuration of the cages was changed once a week. That is, the location of some of the objects that made up the enriched environment was changed and some of them were replaced ${ }^{11}$.

.2) Standard Environment (S) - the animals were kept permanently in the standard bioterium cages, with dimensions of $51 \times 35.5 \times 18.5 \mathrm{~cm}$. They constituted the control animals of the EE group.
At the end, the following experimental groups were formed: PILO+EE $(n=6)$; PILO+S $(n=7)$; $S A L+E E(n=6) ; S A L+S(n=7)$.

At 67 days of age, each animal was submitted to CSD recording for 4 hours. The animals were anesthetized, intraperitoneally, with a mixture of $10 \%$ urethane and $0.4 \%$ alpha chloralose, at a dose of $1000 \mathrm{mg} / \mathrm{kg}$ and $40 \mathrm{mg} / \mathrm{kg}$ (both from Sigma Co., USA), respectively. A tracheal cannula was inserted to facilitate breathing. The animal's rectal temperature was maintained at $36.5 \pm 1.05 \stackrel{\circ}{\circ}$ throughout the experiment. The animal's head was fixed in a stereotactic apparatus to allow the incision and removal of the skin and periosteum and the exposure of the skull. Through trepanation, three holes were made (anterior, middle and posterior, of approximately 2 to $4 \mathrm{~mm}$ in diameter each), aligned parallel to the midline at the level of the right cerebral hemisphere. Two holes were drilled in the parietal bone to record CSD and the other in the frontal bone for its deflagration.

In order for CSD to be provoked, a stimulus was applied (solution of $2 \% \mathrm{KCl}$ soaked in cotton), for 1 minute, every 20 minutes, to the anterior hole. The records of slow voltage variation, which accompanies the phenomenon, were obtained at the two parietal cortical points (one in each orifice). Unpolarizable electrodes (type "Ag$\mathrm{AgCl}$ ") were used, produced in the laboratory itself, against a common reference electrode placed on the nasal bones, where the potential is invariable. Electrodes were connected to a digital data acquisition system (EMG System, São Paulo, Brazil) coupled to a computer was used. At the end of the registration session, each animal, still under deep anesthesia, was euthanized through a bulbar injury.

Statistical analysis was performed using ANOVA, as well as the Holm-Sidak post-test, considering $\mathrm{p}<0.05$ as significant.

\section{RESULTS}

Regarding the speed of CSD spread, the following values were found, expressed as mean $\pm S D$, for the groups $S A L+S, S A L+E E, P I L O+S$, 
PILO+EE, respectively: $3.57 \pm 0.62 \mathrm{~mm} / \mathrm{min}$, $2.96 \pm 0.45 \mathrm{~mm} / \mathrm{min}, 3.03 \pm 0.42 \mathrm{~mm} / \mathrm{min}, 3.30$ $\pm 0.55 \mathrm{~mm} / \mathrm{min}$. ANOVA showed a statistically significant difference between the groups ( $F$ $[3,313]=23,847 ; P<0,001)$ (Figure 1). The Holm-Sidak test showed that animals in the SAL+EE, PILO+S, PILO+EE groups showed a reduction in CSD speed when compared to the control group $(S A L+S)$. In addition, the PILO+EE group showed a slight increase in CSD speed when compared to the PILO+S group.

Regarding the duration of the cortical spreading depression, the following values were found, expressed as mean $\pm S D$, for the experimental groups $\mathrm{SAL}+\mathrm{S}, \mathrm{SAL}+\mathrm{EE}, \mathrm{PILO}+\mathrm{S}, \mathrm{PILO}+\mathrm{EE}$, respectively: $107.18 \pm 16$, $85 \mathrm{~s} ; 96.39 \pm 21.14 \mathrm{~s}$; $129.11 \pm 30.12 \mathrm{~s} ; 93.03 \pm 21.15 \mathrm{~s}$ (Figure 2). ANOVA demonstrated a statistically significant difference between the groups $(F[3,431]=$ 56,408; $P<0,001)$. The Holm-Sidak post-test demonstrated that the PILO+S group showed an increase in the duration of CSD, when compared to the control group. The SAL+EE and PILO+EE groups demonstrated a reduction in the duration of CSD, when compared with the $S A L+S$ and $\mathrm{PILO}+\mathrm{S}$ groups, respectively. However, there were no differences between them.

Regarding the CSD amplitude parameter, the following values were found, expressed as mean $\pm S D$, for the experimental groups $S A L+S$, SAL+EE, PILO+S, PILO+EE, respectively: 5.14 $\pm 1.00 \mathrm{mV}, 6.43 \pm 2.29 \mathrm{mV}, 7.00 \pm 1.64 \mathrm{mV}, 5.43$ $\pm 1.77 \mathrm{mV}$ (Figure 3). ANOVA showed statistically significant difference between the groups $(F[3.404]=25.712 ; \mathrm{P}<0.001)$.

\section{DISCUSSION}

This study addressed, for the first time, the influence of the enriched environment in rats submitted to status epilepticus, using CSD for this purpose. It's possible to observe that both environmental enrichment and pilocarpine, alone, were able to reduce the speed of the spread of CSD. These findings are corroborated by the literature ${ }^{4,12,13}$. However, the causes for this reduction are distinct.
In fact, it has been shown that as brain excitability increases, CSD propagation speed increases. However, this occurs up to a certain limit, from which additional increases in cortical excitability hinder the spread of CSD in the nervous system ${ }^{14,15}$. The epilepticus status induced by pilocarpine markedly increases cortical excitability ${ }^{16}$ and causes brain changes ${ }^{6}$.

Shortly after pilocarpine application, the first excitotoxic damage caused by this alkaloid can be observed, at the morphological level, in the superficial layers of several organs. For example, the endopiriform nucleus, hippocampus hilum, claustrum, piriform cortex and dentate gyrus are affected. Subsequently, the damage is intensified in these areas and spreads to other regions, such as tonsillar nuclei, subiculum, bed of the terminal striated nucleus, entorhinal cortex and ventromedial nucleus of the hypothalamus. This insult can continue to cause damage for weeks and even months, reaching other areas of the brain, such as the thalamus ${ }^{5,6,16}$.

At the neurochemical level, it is reported that the pilocarpine-induced events are capable of generating changes in the ionic gradient across the membrane, changes in the expression of genes for trophic factors, receptors, enzymes and proteins of the cytoskeleton and the extracellular matrix ${ }^{6}$. These authors also mention that the hippocampus of rats submitted to the pilocarpine model shows a reduction in the rate of use of dopamine and an increase in the rate of use of norepinephrine, as well an increase in the glutamate release.

Glutamate activates N-methyl-D-aspartate (NMDA) receptors. When activated, NMDA receptors increase intracellular concentrations of $\mathrm{Ca}^{2+}$ and this activates proteases, nucleases and lipases that cause the cell to undergo necrosis and / or apoptosis ${ }^{6}$. Additionally, pilocarpine induces an increase in the production of reactive oxygen species, contributing to neurodegeneration ${ }^{17}$. All of the cellular damage described above seems to be related to impairments in the spread of CSD. 
Environmental enrichment, in turn, also has a brain level, especially in areas of the major influence on brain morphology, hippocampus ${ }^{18}$. An increase in dendritic neurochemistry and physiology ${ }^{10}$. However, in ramifications of some neuronal populations and contrast to the TLE model with pilocarpine, EE is neurogenesis in the dentate gyrus and in other capable of generating beneficial actions at the areas of the hippocampus is reported ${ }^{1,10}$.

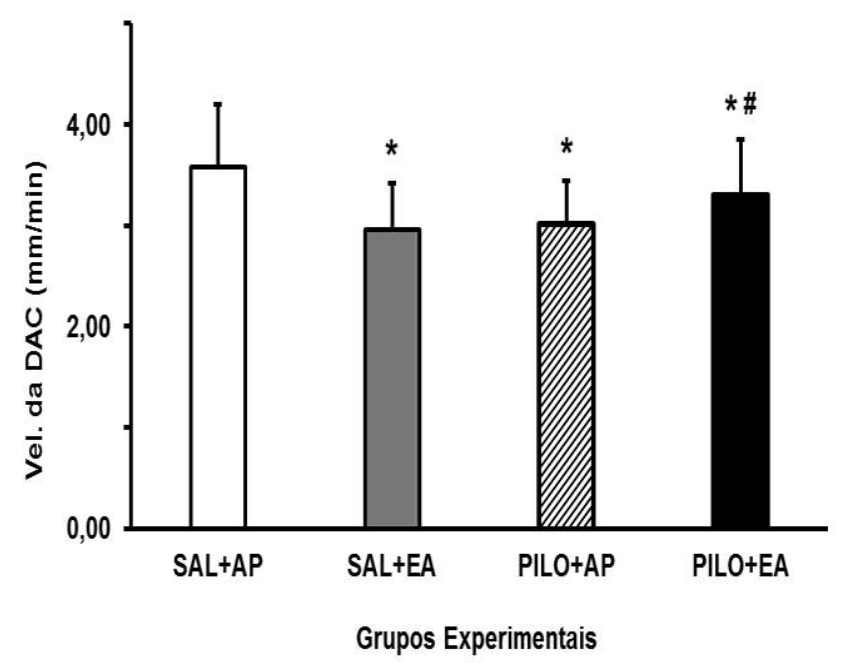

Figure 1 - CSD propagation speed, expressed in $\mathrm{mm} / \mathrm{min}$, between the different experimental groups. The data are expressed as mean \pm SD. The distinct symbols represent statistically significant differences. * $p<0.05$ in relation to the PILO+S group.

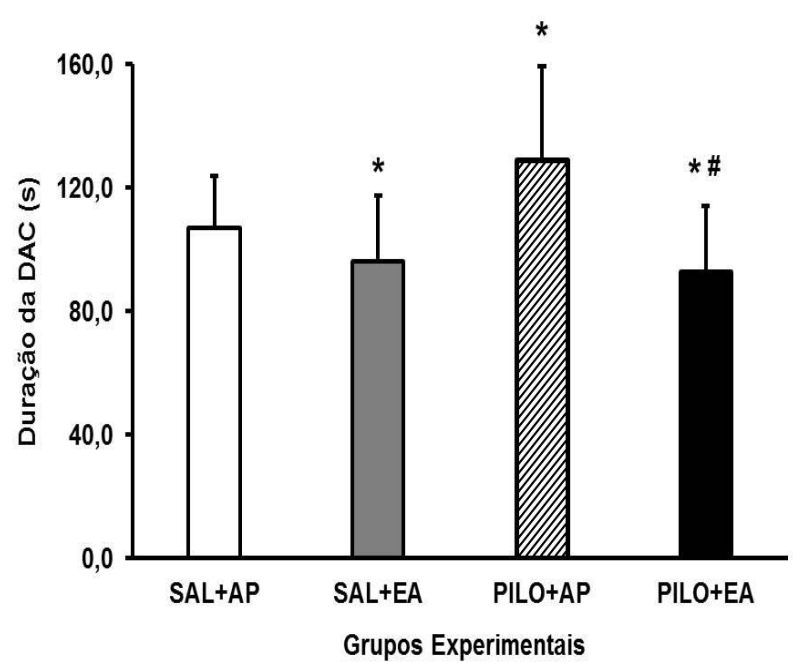

Figure 2 - Duration of CSD, expressed in seconds, between the different experimental groups studied. The data are arranged in mean \pm SD. The distinct signs indicate statistically significant differences. ${ }^{*} p<0.05$ in relation to the $S A L+S$ group. $\# p<0.05$ in relation to the $\mathrm{PILO}+\mathrm{S}$ group

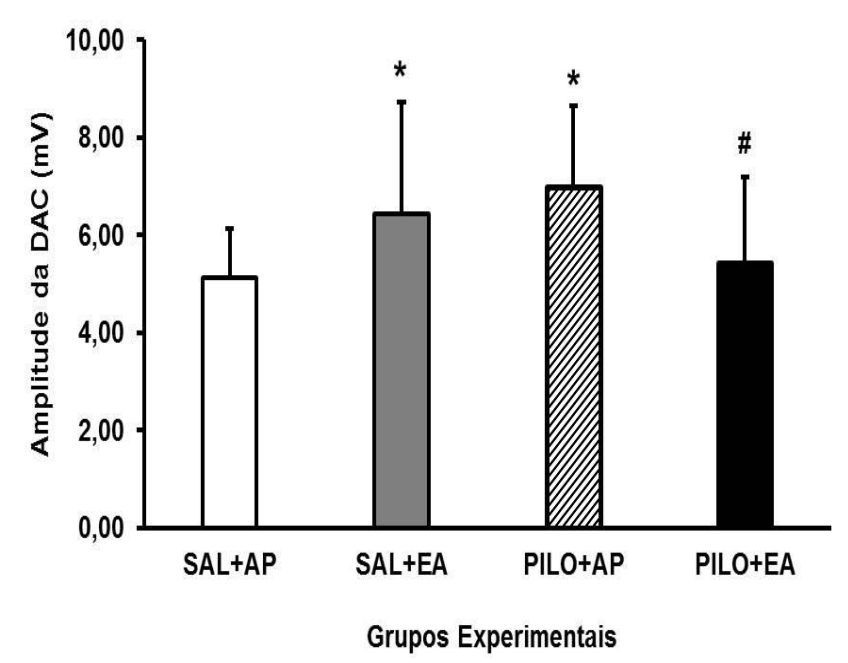

Figure 3 - Amplitude of CSD, expressed in $\mathrm{mV}$, between the different experimental groups. The data are arranged in mean \pm SD. The distinct signs indicate statistically significant differences. ${ }^{*} p<0.05$ in relation to the $S A L+S$ group. \# $p$ $<0.05$ in relation to the SAL+EE and PILO+S groups.

In addition to these changes, EE also causes an increase in the expression of neurotrophins, such as vascular endothelial growth factor (VEGF), brain-derived neurotrophic factor (BDNF) and nervous growth factor (NGF). It also causes changes in the expression of synaptic proteins and NMDA and AMPA (a-amino-3hydroxy-5-methyl-4-isoxazoleproprionic acid) receptors, which are linked to glutamatergic 
signaling, indicating an increase in synaptic plasticity and strength synaptic ${ }^{1}$.

In relation to oxidative processes, it is described that $E E$ is able to attenuate oxidative stress and strengthen antioxidant protection mechanisms ${ }^{19,20}$. Another mechanism reported in the literature suggests that EE stimulates the serotonergic system and an increase in brain serotonin levels is related to a reduction in the speed of the spread of CSD ${ }^{21}$.

Additionally, the EE is linked to the promotion of physical activity, since the cages are larger, there is the presence of ramps, tunnels and objects that can be moved; that is, the EE stimulates physical practice4. Evidence shows that physical exercise considerably improves brain function ${ }^{21}$. Exercise promotes an increase in late hippocampal neurogenesis, synaptic plasticity and improves cerebral vascularity ${ }^{1}$. In addition, it favors the release of neurotrophins and the balance of neurotransmitter levels ${ }^{22}$. All of these beneficial brain changes reduce cortical excitability and consequently the rate of CSD spread.

In the present work, it is possible to observe that the speed of CSD spread in the PILO+EE group was statistically lower than in the control group $(S A L+S)$, however higher than in the PILO+S group, that is, the EE was able to reduce brain excitability caused by status epilepticus, probably due to the addition of the mechanisms described previously, however, it was not enough to reach the $S A L+S$ group. .

Regarding the duration parameter of the electrophysiological phenomenon, it was possible to observe an increase in the duration of CSD in the PILO+S group compared to the control group $(S A L+S)$. Data have shown that this is related to an increase in the formation of free radicals, induced by pilocarpine ${ }^{17}$, and to a higher difficulty in metabolic recovery ${ }^{23}$.

On the other hand, both groups submitted to EE showed reduced duration of CSD when compared to their controls in a standard environment. Moreover, when submitted to EE, the pilocarpine group did not differ from the saline group. This once again indicates that environmental enrichment had a neuroprotective effect, attenuating the damage caused by oxidative stress ${ }^{20}$, which is normally induced by pilocarpine ${ }^{17}$. These results reinforce the hypothesis that EE improves the metabolic response of the cerebral cortex during CSD.

Concerning the amplitude, the pilocarpine group maintained in a standard environment showed the greatest amplitude when compared to the control group $(S A L+S)$. This strengthens the previous findings of this study that show that pilocarpine increases cortical excitability ${ }^{16}$. Surprisingly, an increase in the amplitude of CSD was observed in animals submitted to EE $(S A L+E E)$. Further studies are needed to confirm this phenomenon, which was not observed by another study4. In contrast, EE reduced the amplitude of the pilocarpine group, making it statistically similar to the control group $(S A L+S)$. This reinforces our findings of CSD propagation speed that the $\mathrm{EE}$ is able to improve the neural circuitry and decrease the hyperexcitability caused by pilocarpine.

\section{CONCLUSION}

Environmental enrichment reduced the cortical excitability caused by pilocarpine-induced status epilepticus. This fact is believed to be related to the ability of $E E$ to promote antioxidant effects at the brain level, induce neurogenesis and improve the brain's excitatory/inhibitory balance related to glutamate and serotonin.

The topic addressed in this study is of great relevance, as it proposes that the improvement, even at low cost, of environmental conditions can favor the treatment of epilepsies, such as temporal lobe epilepsy. Additionally, this study motivates future investigations, in which further enrichment of the environment could be analyzed. For example, olfactory stimulation could be added. It is possible that an environment with more stimuli promotes an even greater reduction in cortical excitability, which is exacerbated in epilepsies. 
We thank the Foundation for the Support of Science and Technology of the State of Pernambuco (FACEPE) for the financial support to carry out this research and the Laboratory of Electroneurophysiology - UFPE for providing the support to be able to carry out this study.

\section{REFERENCES}

1. Nithianantharajah J, Hannan A. Enriched environments, experience-dependent plasticity and disorders of the nervous system. Nature Reviews Neuroscience. 2006;7(9):697-709.

2. Mármol $F$, Sánchez J, Torres $M$, Chamizo V. Environmental enrichment in the absence of wheel running produces beneficial behavioural and anti-oxidative effects in rats. Behavioural Processes. 2017;144:66-71.

3. Ohline S, Abraham W. Environmental enrichment effects on synaptic and cellular physiology of hippocampal neurons. Neuropharmacology. 2019;145:3-12.

4. Alves D, Sousa M, Tavares M, Santos L, Batistade-Oliveira-Hornsby M, Amancio-dos-Santos A. Environmental enrichment reduces brain excitability in adult rats overnourished during lactation. Arquivos de Neuro-Psiquiatria. 2019;77(8):555-559.

5. Fernandes M. Epilepsia do lobo temporal: mecanismos e perspectivas. Estudos Avançados. 2013;27(77):85-98.

6. Scorza F, Arida R, Naffah-Mazzacoratti M, Scerni D, Calderazzo L, Cavalheiro E. The pilocarpine model of epilepsy: what have we learned?. Anais da Academia Brasileira de Ciências. 2009;81(3):345-365.

7. Kinjo, E. R. Conexinas na epilepsia experimental induzida por pilocarpina: abordagem molecular e eletrofisiológica [tese]. São Paulo: Instituto de Ciências Biomédicas; 2011.

8. Somjen G. Aristides Leão's discovery of cortical spreading depression. Journal of Neurophysiology. 2005;94(1):2-4.

9. Gryglas A, Smigiel R. Migraine and Stroke: What's the Link? What to Do?. Current Neurology and Neuroscience Reports. 2017;17(3).

10. Vrinda M, Sasidharan A, Aparna S, Srikumar B, Kutty B, Shankaranarayana Rao B. Enriched environment attenuates behavioral seizures and depression in chronic temporal lobe epilepsy. Epilepsia. 2017;58(7):1148-1158.

11. Sampedro-Piquero $P$, Zancada-Menendez $C$, Begega A, Rubio S, Arias J. Effects of environmental enrichment on anxiety responses, spatial memory and cytochrome c oxidase activity in adult rats. Brain Research Bulletin. 2013;98:19.

12. Guedes R, Cavalheiro E. Blockade of spreading depression in chronic epileptic rats: reversion by diazepam. Epilepsy Research. 1997;27(1):33-40.

13. Santos L. G. C. e Amancio-dos-Santos A. O efeito nootrópico sobre a depressão alastrante cortical: um possível mecanismo de ação antiepiléptica. Recife: Relatório (Iniciação Científica), Departamento de Fisiologia e Farmacologia, Universidade Federal de Pernambuco, 2019.

14. Leo L, Gherardini L, Barone V, De Fusco M, Pietrobon D, Pizzorusso $T$ et al. Increased Susceptibility to Cortical Spreading Depression in the Mouse Model of Familial Hemiplegic Migraine Type 2. PLoS Genetics. 2011;7(6):e1002129.

15. Vitor-de-Lima S, Medeiros L, Benevides R, dos Santos C, Lima da Silva N, Guedes R. Monosodium glutamate and treadmill exercise: Anxiety-like behavior and spreading depression features in young adult rats. Nutritional Neuroscience. 2017;22(6):435-443.

16. Nogueira R, Pessoa D, da Silva E, Costa E. Can a hypercholesterolemic diet change the basal brain electrical activity and during status epilepticus in rats?. Metabolic Brain Disease. 2018;34(1):71-77.

17. Pestana R, Kinjo E, Hernandes M, Britto L. Reactive oxygen species generated by NADPH oxidase are involved in neurodegeneration in the pilocarpine model of temporal lobe epilepsy. Neuroscience Letters. 2010;484(3):187-191.

18. Auvergne R, Leré $C$, El Bahh $B$, Arthaud $S$, Lespinet $V$, Rougier $A$ et al. Delayed kindling epileptogenesis and increased neurogenesis in adult rats housed in an enriched environment. Brain Research. 2002;954(2):277-285.

19. Herring $A$, Blome $M$, Ambrée $O$, Sachser $N$, Paulus W, Keyvani K. Reduction of cerebral oxidative stress following environmental enrichment in mice with Alzheimer-like pathology. Brain Pathol. 2020;20(1):166-175.

20. Muhammad $M$, Magaji $R$, Mohammed $A$, Isa $A$, Magaji $M$. Effect of resveratrol and environmental enrichment on biomarkers of oxidative stress in young healthy mice. Metabolic Brain Disease. 2016;32(1):163-170.

21. Monteiro H, Barreto-Silva N, Santos G, Santos A, Sousa M, Amâncio-dos-Santos Â. Physical exercise versus fluoxetine: Antagonistic effects on cortical spreading depression in Wistar rats. European Journal of Pharmacology. 2015;762:49-54.

22. Jacotte-Simancas A, Costa-Miserachs D, CollAndreu M, Torras-Garcia M, Borlongan C, PortellCortés I. Effects of Voluntary Physical Exercise, 
Roberto Carlos da Silva Bandeira et al., IJNR, 2021; 5:13

Citicoline, and Combined Treatment on Object

Recognition Memory, Neurogenesis, and Neuroprotection after Traumatic Brain Injury in

Rats. Journal of Neurotrauma. 2015;32(10):739751.
23. Sonn J, Mayevsky A. Responses to Cortical Spreading Depression under Oxygen Deficiency. The Open Neurology Journal. 2012;6(1):6-17. 